\title{
Digital Grey Scale Image Enhancement based on Anisotropic Non-Linear Diffusion Technique
}

\author{
Honey Saxena \\ Jaipur National University \\ Jaipur, India
}

\author{
Kalyan Acharjya \\ Jaipur National University \\ Jaipur, India
}

\begin{abstract}
The era of image processing has influenced every part of the universe right from medical field to space research. Digital image enhancement is the process to improve quality metrics of a noisy image. In this presented paper based on anisotropic non-linear diffusion technique to enhance the input noisy gray scale images, here mainly focused on speckle noise, which aremainly cause by today's modern image processing sensing devices. The value of quality matrices of an enhanced image are PSNR $10 \mathrm{~dB}$ and SNR $11 \mathrm{~dB}$. After comparison the values of image parameters with different technique, the presented work not only attained the better result but also itreduce the complexity of digital circuit as its fall under spatial domain category. Finally the paper concluded that anisotropic nonlinear diffusion technique is efficient forgrey scale image enhancement, especially for reduction of speckle noise.
\end{abstract}

\section{Keywords}

Image Enhancement, Anisotropic, Diffusion, PSNR etc.

\section{INTRODUCTION}

An image is a two dimensional light intensity function. Digital image enhancement is the process to improve a noisy or distorted image for visualization point of view or improved quality parameters of an image. Image enhancement broadly in two categories: one is spatial domain which purely deals with pixel relation and second one is frequency domain which deals on frequency spectrum. The work was carried on MATLAB tool.

Image noise is the random variation of brightness. There are different types of noises, say salt and pepper noise, gaussian noise or white noise, speckle noise etc. The reduction of spackle noise is challenging in today's digital world, because it mainly due to the sensing circuits incorporated with camera or digital image processing devices.

\section{TECHNIQUE FOR IMAGE ENHANCEMENT}

There are broadly following categories [4] of techniques are available for achieve desired enhancement from noisy images.

\subsection{Spatial Domain Method}

This method totally deals with the pixel values of given image to achieve desired enhancement. The whole operations directly related with pixel values.

\subsection{Frequency Domain Method}

In this technique, operations are performed on the Fourier transform or any other transform methods which are used enhanced the input image.

The sub-categories of image enhancement techniques are as follows:
2.2.1 Histogram Modification: It is the effective technique by analysis the intensity variations of input noisy image and performs the desired mechanism to improve the quality metrics of a given image.

2.2.2 Filtering: In the filtering technique, intensity of one pixel is combined with the intensity of neighboring pixels to enhance the image; it may be linear and nonlinear filter.

2.2.3 Homomorphic Filtering: It normalizes the brightness across an image and increase contrast of given image.

2.2.4 Edge Enhancement: It enhances the edge contrast of an image not only increase sharpness of input image but also detect discontinuities in gray level.

\section{PERONA MALIK ANISOTROPIC NON-LINEAR DIFFUSION IMAGE ENHANCEMENT TECHNIQUE}

Anisotropic diffusion also called Perona Malik diffusion is a technique for reducing image noise without removing fundamental parts of the image content. This technique's fundamental concept belongs to image edges, i.e. sustained changes in image intensity. The extraction of edges from a digital image allows a valuable abstraction of information and a reduction in processing and storage costs also.On the other hand, in anisotropic diffusion is a linear low pass filtering process which is not able to preserve the position of important edges through the scale space. Anisotropic diffusion meets criteria and has been used effectively in conjunction with edge detection [2]. It preserves edges through the image smoothing process. It yields intraregional smoothing not interregional smoothing by restart diffusion at the image edges.

The function that prevents smoothingat the edges is the diffusion coefficient [1], i.e.it is the most critical step in performing diffusion based edge detection.

$$
\mathrm{I}_{\mathrm{t}}=\operatorname{div}(\mathrm{c}(\mathrm{x}, \mathrm{y}, \mathrm{t}) \nabla \mathrm{I})=\mathrm{c}(\mathrm{x}, \mathrm{y}, \mathrm{t}) \Delta \mathrm{I}+\nabla \mathrm{c} \nabla \mathrm{I}
$$

"I" belongs to the family of gray level and "div" is the divergence operator $\Delta$ respectively. The Laplacian operatorZand gradientwith respect to space variables, $\mathrm{c}(\mathrm{x}, \mathrm{y}, \mathrm{t})$ is the diffusion coefficient, which controls the rate of diffusion and is usually chosen as a function of the image gradient so as to preserve edges in the image (smoothing).Gradient of the brightness function tells if $\mathrm{x}, \mathrm{y}$ is a part of edge or not and where gradient is high, on that point smoothing is occur. On the other side if it is low than less smoothing occur. Then we can find the value of proposed [1] two diffusion coefficients using equation 2 ( $2 \mathrm{a}$ and $2 \mathrm{~b}$ ). 


$$
\begin{aligned}
& c(\|\nabla\|)=e^{-\left(\frac{\|\nabla \nabla\|}{K}\right)^{2}} \\
& c(\|\nabla\|)=\frac{1}{1+\left(\frac{\|\nabla I\|}{K}\right)^{2}}
\end{aligned}
$$

Function "c" controls blurring intensity according to magnitude of gradient of brightness. " $k$ " controls the sensitivity to edges and intensity. If $\mathrm{k}$ is less, then conduction occurs across step edges, otherwise $\mathrm{k}$ reduces the modification of intensity gradient on conduction. In these diffusion operations using equations 3 has the ability to sharpen edges .The most interesting fact of anisotropic diffusion is that the enhancement of high contrast edges with the help of the intensity gradient into the intensity difference of a pixel to its four neighbors as the equations 3 in all four directions (north, south, east and west).

$$
\begin{gathered}
\nabla_{N} I_{i, j}=I_{i-1, j}-I_{i, j} \\
\nabla_{S} I_{i, j}=I_{i+1, j}-I_{i, j} \\
\nabla_{E} I_{i, j}=I_{i, j+1}-I_{i, j} \\
\nabla_{W} I_{i, j}=I_{i, j-1}-I_{i, j}
\end{gathered}
$$

N, S, E, W mnemonic subscripts for north, south, east, west directions. $\nabla$ is the nearest neighbor difference. Iterating this scheme can be considered as moving towards coarser resolutionsin scale space dimensions [1]. The conduction coefficients are updated in each iteration according to the function of the brightness gradient, which calculated based on the fourdimensional components of the diffusion constant as per equations 4 .

$\mathrm{C}_{\mathrm{N}}(\mathrm{x}, \mathrm{y})=\mathrm{g}\left((\nabla \mathrm{I})_{\mathrm{N}}(\mathrm{x}, \mathrm{y})\right)$
$\mathrm{C}_{\mathrm{S}}(\mathrm{x}, \mathrm{y})=\mathrm{g}\left((\nabla \mathrm{I})_{\mathrm{S}}(\mathrm{x}, \mathrm{y})\right)$
$\mathrm{C}_{\mathrm{E}}(\mathrm{x}, \mathrm{y})=\mathrm{g}\left((\nabla \mathrm{I})_{\mathrm{E}}(\mathrm{x}, \mathrm{y})\right)$
$\mathrm{C}_{\mathrm{W}}(\mathrm{x}, \mathrm{y})=\mathrm{g}\left((\nabla \mathrm{I})_{\mathrm{W}}(\mathrm{x}, \mathrm{y})\right)$

After the iterations completed the pixel intensity calculated using equation [1].

$I_{i, j}^{t+1}=I_{i, j}^{t}+\lambda\left[C_{N} \nabla_{N} I+C_{S} \nabla_{S} I+C_{E} \nabla_{E} I+C_{W} \nabla_{W} I\right]_{i, j}^{t}$

Where $\lambda$ used for better stability (no blurred). From the equation 5 each pixel gives the desired enhancement of the input noisy image. As seen the throughout technique, there is no need to transform an image into another domain. It is the very easy way to remove the noise from the input image to achieve the quality image of a gray scale image.

The main algorithm based on smoothing algorithm having following criteria.

1. Causality: No fake details should be added when image passing from finer to coarser level.

2. Immediate Localization: At each resolution the region boundary should be sharp.

3. Piecewise smoothing: Intraregional smoothing should be done over interregional smoothing andintraregional smoothing diffusion done within the region or image boundary.

The smoothing algorithm is represents the criteria should get better results.As anisotropic nonlinear diffusion technique is incorporated all criteria of smoothing algorithm used for enhancement the noisy image.

\section{RESULT ANALYSIS}

The result of image enhancement based on non-linear anisotropic diffusion technique is shown in the Fig 2. The quality matrices of an image determined the quality of an image. As following quality matrices are mainly used for determine the quality of a distorted or lossy compression image as PSNR (Peak signal power to noise power ratio), SNR (Average signal power to noise power), MSE (Mean square error) and RMSE (Root mean square error which measure the differences between the desired distribution and actual distribution for each targeted population segment and calculate a weighted average error) etc. For compare the presented work result, the authors also included the image enhancement using median filter, which are shown in Fig 4.

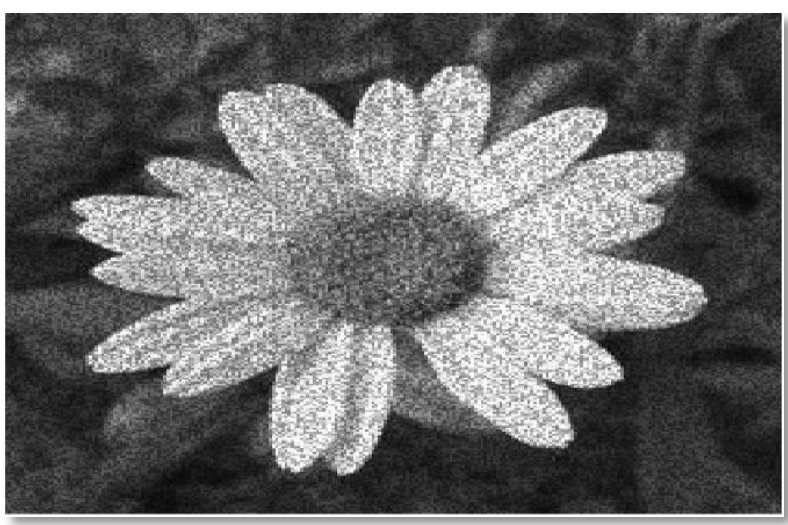

Fig 1: Input Image with Speckle Noise

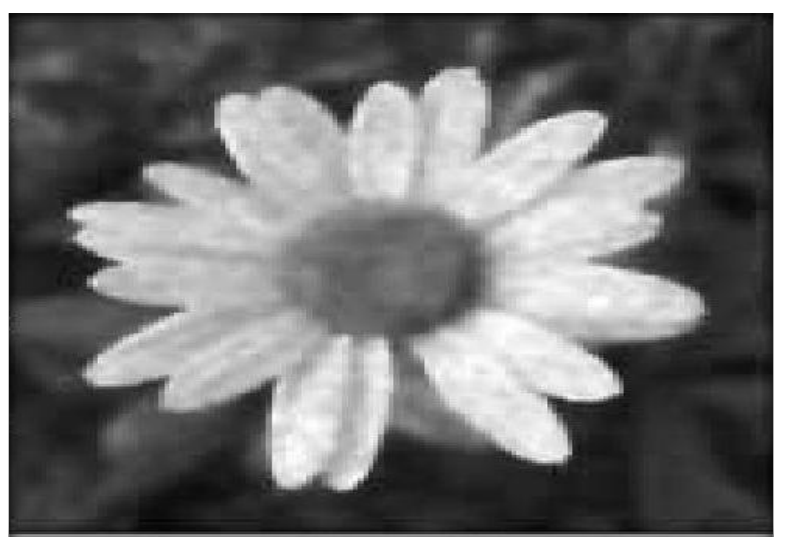

Fig 2: Output Enhanced Image

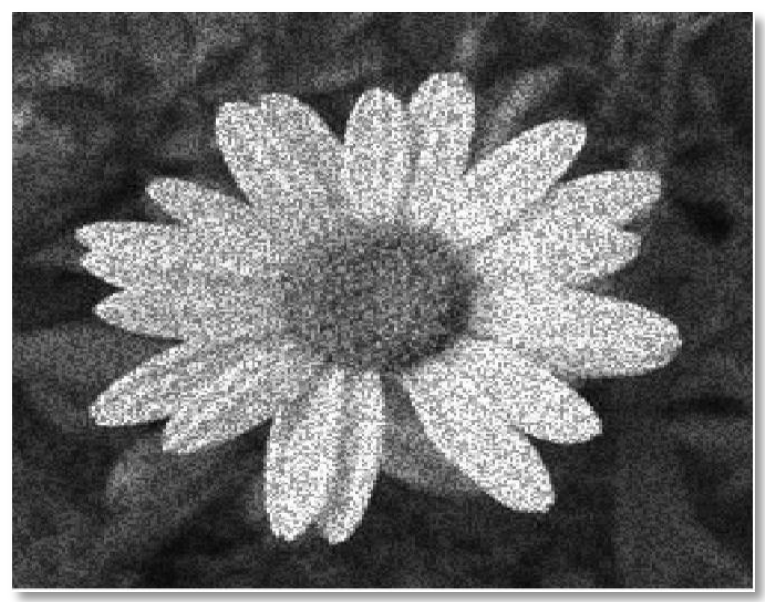

Fig 3: Input Image with Speckle Noise 


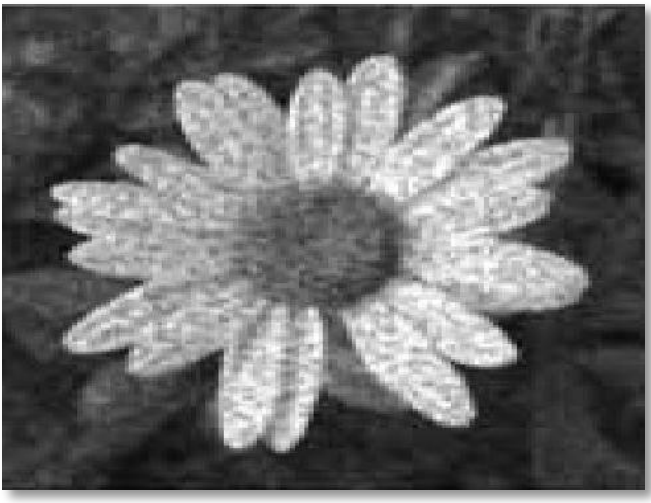

Fig 4: Output Enhanced Image

The comparison result of different techniques is presented in Table 1. In all simulation results are based on same input noisy image.

Table 1: Comparison of result in different Image enhancement technique

\begin{tabular}{|l|l|l|l|l|}
\hline $\begin{array}{l}\text { Quality } \\
\text { Metrics }\end{array}$ & $\begin{array}{l}\text { Presented } \\
\text { Work }\end{array}$ & \multicolumn{4}{|l|}{$\begin{array}{l}\text { Used inbuilt Matlab function or } \\
\text { general algorithm for following } \\
\text { Filters and Referred paper [5] }\end{array}$} \\
\cline { 2 - 5 } & Diffusion & $\begin{array}{l}\text { Median } \\
{[6]}\end{array}$ & $\begin{array}{l}\text { Kernel } \\
{[5]}\end{array}$ & $\begin{array}{l}\text { Weiner } \\
{[6]}\end{array}$ \\
\hline PSNR & 10 & 8 & 8.5 & 7 \\
\hline MAE & 25 & 26.15 & 26 & 10.7 \\
\hline RMSE & 14 & 12 & 12.5 & 10 \\
\hline SNR & 11 & 5 & 9 & 8 \\
\hline
\end{tabular}

As PSNR is most important parameter for determine the quality of an enhanced image. From the Table 1, it justified that anisotropic non-lineardiffusion technique is better as comparison to other similar parallel technique. The result attained the PSNR and SNR are 10 and $11 \mathrm{~dB}$ respectively. In the Table 1 also included the result values of the referred paper [5], which worked based on Median and Weiner filter.

The comparison result of PSNR and SNR of same enhanced image in different techniques are shown in bar diagram as Fig 5 and Fig 6 respectively.

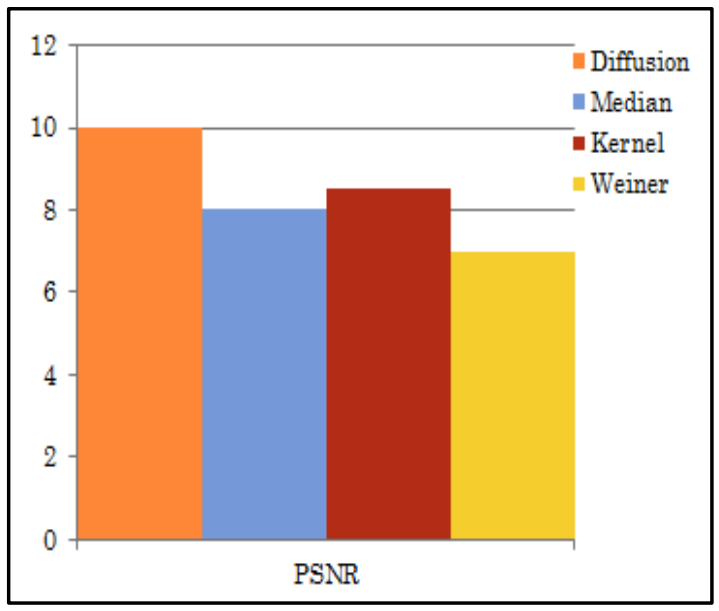

Fig 5: PSNR Comparison

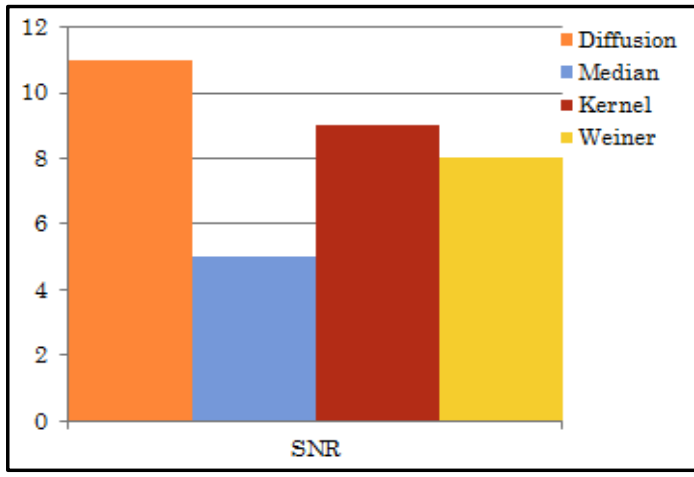

Fig 6: SNR Comparison

\section{CONCLUSION AND FUTURE WORK}

Noise can appear in image from a variety of sources during the acquisition process, illumination variations, due to quality resolution of cameras and during processing of imageetc. As modern world urged for efficient noise removing technique for digital image processing or computer vision, which arewide spread everywhere. This presented work not onlyshowed the efficient image enhancement technique in spatial domain but also quite simple as compare with transform in frequency domain. This may also reduce the complexity of digital circuit when it will be implemented it. The enhanced results justifies that non-linear anisotropic diffusion technique is far better than other technique, especially in case removing the speckle noise from a distorted image. The future work will be tried to model all three planes of RGB image with nonlinear anisotropic diffusion technique.

\section{REFERENCES}

[1] P. Perona and J. Malik,"Scale-Space and Edge Detection Using Anisotropic Diffusion”, IEEE Transactions on Pattern analysis and Machine Intelligence Vol.12.No.7,July1990.

[2] Eric J. Wharton, Karen A.Panetta, SosS.Agaian, "Edge Preserving Image Enhancement Using Anisotropic Diffusion", Proc. SPIE 6812, Image Processing: Algorithms and Systems VI, 681218 March 03, 2008; doi:10.1117/12.766893.

[3] Marius Lysakar, ArvidKundervold and Xue-ching Tai,"Noise Removal using fourth order Partial differential Equations with applications to medical Magnetic Resonance Images in Space and Time”, IEEE Transactions on Image Processing, Vol.12, No.12 Dec2003.

[4] Rafael C. Gonzalez, Richard E. Woods, "Digital Image Processing", $3{ }^{\text {rd }}$ edition, Pearson Publication, ISBN-139788131726952

[5] Jang, Dae, Jong, Jiwon, "Measurement of image quality in CT images reconstructed with different kernels", Journal of the Korean Physical Society, Vol. 58, No. 2, February 2011, pp. $334 \notin 342$.

[6] Ashok Kumar, Manoj Gupta, Suresh Kumar, "Performance Comparison in Weiner and Median filter in image denoising", International Journal of Computer Applications. 0975-8887, Vol.12, No.4, Nov 2010.

[7] Jean-Luc Starck, Fionn Murtagh, Emmanuel J. Candès, and David L. Donoho, "Gray and Color Image Contrast Enhancement by the Curvelet Transform", IEEE Transactions on Image Processing, Vol.12, No.6, June 2003. 